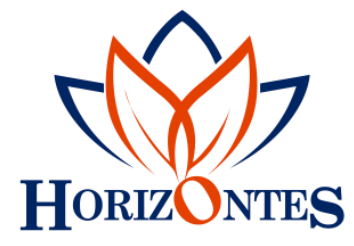

www.revistahorizontes.org

\title{
Resiliencia educativa: Influencia en la lectoescritura en niños con estado de vulnerabilidad de EGB. Media
}

\author{
Educational Resilience: Influence on literacy in children with vulnerability state of GBS, \\ Medium
}

\section{Resiliência educacional: Influência na alfabetização em crianças com um estado vulnerável de GBS. Metade}

ARTICULO DE REVISIÓN

\author{
Jenny Karina Palate Chango \\ jenny.k.palate.c@pucesa.edu.ec \\ ORCID: 0000-0001-6235-9316
}

\author{
Elena del Rocío Rosero Morales \\ erosero@pucesa.edu.ec
}

ORCID: 0000-0001-7004-3207

\section{Pontificia Universidad Católica del Ecuador-Sede Ambato, Ecuador}

Recibido 03 de febrero 2021 | Arbitrado y aceptado 15 de marzo 2021 | Publicado en marzo 2021

\section{RESUMEN}

Durante los últimos tiempos el bajo nivel económico, el escaso acceso a la salud y educación bajo condiciones adversas de vulnerabilidad son factores externos que dificultan la capacidad del estudiante en la educación, procesamiento de información, capacidad de análisis, síntesis y comprensión lectora, los objetivos propuestos son; fundamentar teóricamente a la resiliencia educativa como influencia en la lectoescritura en niños con estado de vulnerabilidad de EGB. Media, para determinar cómo incide la resiliencia educativa en la lectoescritura en niños y relacionar a la resiliencia educativa como influencia en la lectoescritura en niños en estado de vulnerabilidad, bajo la modalidad de investigación de tipo cualitativa al describir de forma narrativa estudios bibliográficos; de entre las conclusiones se obtiene que para procesos de lectoescritura de los niños se deben presentar óptimas condiciones escolares, familiares y emocionales, afectado más aun a niños que por factores biopsicosociales se encuentran inestables sin resiliencia educativa por encontrarse en vulnerabilidad.

Palabras clave: Resiliencia; lectura-escritura; enseñanza, vulnerabilidad

\section{ABSTRACT}

In recent times, the low economic level, the scarce access to health and education under adverse conditions of vulnerability are external factors that hinder the student's ability in education, information processing, analysis, synthesis and reading comprehension capacity, the objectives proposed are; theoretically base educational resilience as an influence on literacy in children with a vulnerable state of GBS. Medium, to determine how educational resilience affects literacy in children and relate educational resilience as an influence on literacy in children in a state of vulnerability, under the modality of qualitative research when describing bibliographic studies in a narrative way; From among the conclusions, it is obtained that for children's literacy processes, optimal school, family and emotional conditions must be presented, even more affected children who, due to biopsychosocial factors, are unstable without educational resilience because they are in vulnerability.

Key words: Resilience; reading writing; teaching vulnerability 


\section{RESUMO}

Nos últimos tempos, o baixo nível econômico, o escasso acesso à saúde e educação em condições adversas de vulnerabilidade são fatores externos que dificultam a capacidade do aluno em educação, processamento de informação, capacidade de análise, síntese e compreensão leitora, os objetivos propostos são; teoricamente, apoiar a resiliência educacional como uma influência na alfabetização em crianças com um estado vulnerável de GBS. Médio, para determinar como a resiliência educacional afeta a alfabetização de crianças e relacionar a resiliência educacional como uma influência na alfabetização de crianças em estado de vulnerabilidade, na modalidade de pesquisa qualitativa ao descrever estudos bibliográficos de forma narrativa; Das conclusões obtém-se que para os processos de alfabetização das crianças devem ser apresentadas condições ideais de escola, família e emocional, ainda mais crianças afetadas que, devido a fatores biopsicossociais, são instáveis sem resiliência educacional por se encontrarem em vulnerabilidade.

Palavras-chave: Resiliência; leitura/escrita; vulnerabilidade de ensino

\section{INTRODUCCIÓN}

El presente artículo de revisión tiene como prioridad, investigar la resiliencia educativa y su influencia en la lectoescritura, delimitando a la resiliencia "como la capacidad de adaptación de un ser vivo frente a un agente perturbador, un estado o situación adversa"; por lo indicado, en el contexto escolar los estudiantes en riesgo o vulnerabilidad, provienen de un ambiente social inseguro, debido a conflictos familiares, problema de vivienda, escasos recursos económicos, desempleo; factores que aumentan la incertidumbre, pérdida de autoestima, miedo, temor, ansiedad; lo que perjudica en la interacción entre las actitudes, capacidades, el entorno contextual y familiar (Pérez, 2016), expone que, los niños, niñas y adolescentes en riesgo o vulnerabilidad, exteriorizan comportamientos que incluyen la hiperactividad, desatención, tristeza, preocupación y conductas disruptivas evidenciadas en el aula en el bajo rendimiento académico.

Según Pinargote, Cedeño, y Romero (2018) en varias instituciones educativas, se habla de resiliencia como un fenómeno que destaca las capacidades humanas frente a la adversidad, hecho que no resulta casual por la valoración que dan a la autonomía y a los derechos individuales. Se puede afirmar que los estudios han seguido la línea de la psicología positiva, rama para la cual la psique humana, no sólo ayuda a resolver los problemas de salud mental de los individuos, también favorece en una mejor calidad de vida y bienestar. Lamentablemente, la vulnerabilidad social y educativa ha dado origen en la falta de hábito por la lectura, las dificultades en la interpretación de mensajes, elementos que al no ser reforzados deterioran la comprensión lectora, imponiéndose como una actividad mecánica dentro del proceso de aprendizaje. Según Barros (2017), resalta que en las instituciones educativas no se formula prácticas de lectura en los estudiantes, los docentes se dedican a realizar diferentes temas que no aportan en el desarrollo de la escritura en los niños y niñas sobretodo en educación básica, lo que conlleva a una formación con escolares no críticos, no reflexivos, con insuficiente participación, un vocabulario poco fluido y dificultad en la expresión y transmisión de ideas e ideologías.

Desde la visión educativa Eusse y González (2018) afirma que la resiliencia es una capacidad que poseen los seres humanos; sin embargo, requiere de un proceso que incluye el contexto, la familia, la educación y las interacciones entre las otras personas del entorno; elementos que influyen en la lectura y la escritura. Autores como (Camacho, et. al., 2018) hacen hincapié en la implementación de proyectos y programas con la finalidad de 
fortalecer en los educandos las competencias en lectura y escritura son numerosos; en los niveles de educación los estudiantes fortalecen las habilidades para la lectura y escritura; existiendo la posibilidad de que al fortalecer valores transversales como la autonomía, la responsabilidad, la creatividad y la autoestima, pata lograr el aprendizaje de la lectoescritura, el descubrimiento de sus potencialidades, rescatando sus fortalezas y orientándolos en la construcción de proyectos de vida. Según Tomasini, (2012) para construir la resiliencia, la escuela será un ambiente clave que promueva el desarrollo de las capacidades que les permitan sobreponerse a la adversidad, a adquirir las competencias sociales $\mathrm{y}$ académicas beneficiando en la habilidad de leer y escribir adecuadamente; siendo la lectoescritura un proceso en la comprensión del texto, la comunicación y meta cognición; elementos inseparables del proceso mental.

En la provincia de Cotopaxi, según Mora (2018), durante la adquisición de la lectoescritura la mayoría de estudiantes denotan dificultades para desarrollar la capacidad de aprendizaje con la educación tradicional, incrementando las desigualdades que afectan en la integración de los educandos, el desarrollo emocional, social y educativo; la comprensión lectora, las habilidades comunicativas y la capacidad de socialización. Caiza, Morales, y Barragán, (2018), el objetivo principal de la resiliencia es incrementar la capacidad de adaptación y salvaguardar la integridad física de los estudiantes y docentes de cada institución; sin embargo, Gómez, (2017) menciona, en el ámbito educativo la resiliencia es un término utilizado para describir acciones en diferentes situaciones de riesgo que influyen en la seguridad, coordinación, autocontrol, persistencia en el aprendizaje generando afectaciones en la adquisición de habilidades comunicativas, la comprensión lectora, el desarrollo cognitivo; es decir repercute en el desarrollo integral. Para Ortíz, (2016) la ausencia de hábito a la lectura se debe a la falta de estimulación en los hogares, los padres desconocen las actividades educativas que realizan sus hijos; por otro lado en la mayoría de instituciones no fortalecen la destreza en la lectura durante los primeros años de escolaridad provocando el desinterés, lo que genera irritabilidad, cansancio o aburrimiento, lo que conlleva a una escasa comprensión lectora teniendo como consecuencia estudiantes con mala memoria y escaso vocabulario.

En este mismo sentido los niños en situación de desventaja social, familiar o personal y que son sujetos de riesgo de exclusión educativa: fracaso escolar, inadaptación y conflictividad, componentes que influyen en la comprensión lectora; según Alcívar, (2013) lo cual genera problemas en la comprensión lectora y el arte de comunicarse, siendo así que los niños y niñas presentan deficiencias al leer y escribir, los cuales son un indicador de que existe algún déficit que influye en la capacidad de analizar, criticar y reflexionar, siendo necesario que la comunidad escolar, de forma particular el profesorado afronte los nuevos retos de la educación actual y desarrollen dinámicas que contribuyan en la formación de personas capaces de participar activamente en la sociedad

Según Arias (2017), en el contexto educativo de formación, los profesores y escolares tienen la posibilidad de aprender de sus propias experiencias; desde esta visión, la resiliencia es el proceso humano que permite la recuperación positiva del ser humano frente a las adversidades, sin embargo el escaso conocimiento de los maestros en la utilización de actividades, técnicas o estrategias que 
favorezcan en la resiliencia perjudica en la adquisición de habilidades interpersonales, la resolución de conflictos o dificultades; factores que limitan el aprendizaje en base a los errores; además genera una barrera en el alcance de la solidaridad, respeto, conductas de los responsables y transformación positiva de la realidad social.

\section{MÉTODO}

Se elaboró una investigación documental considerando el meta-análisis como método, relacionando la búsqueda con la resiliencia educativa, residencia, entre otros, donde se resaltó las revisiones teóricas de autores precursores para el desarrollo científico del tema. La búsqueda se llevó a cabo mediante las bases de datos de revistas indexadas en donde se consideraron artículos publicados en portales académicos, bibliotecas universitarias, organizaciones educativas, paginas oficiales y gubernamentales nacionales e internacionales, Scielo, Redalyc, entre otras. Amparadas en una revisión descriptiva acerca teorías de la resiliencia educativa y su influencia en la lectoescritura en niños con estado de vulnerabilidad de Educación General Básica Media, siendo de gran utilidad en contextos educativos para procesos de enseñanza $y$ aprendizajes, y también interesará a muchas personas de campos afines a la sociedad, educación y psicología, utilizando como descriptores de búsqueda a la resiliencia educativa, lectoescritura en niños, vulnerabilidad en niños.

\section{DESARROLLO Y DISCUSIÓN}

Para Flores y Marcillo (2020), varios escolares, enfrentan situaciones complejas, algunas estresantes y otras traumáticas, sintiéndose desfavorecidos en el entorno y hasta excluidos socialmente; por lo expuesto, los maestros y maestras considerados entes activos y transformadores de la educación, deben aplicar lecturas motivadoras que estimulen capacidades no innatas en los estudiantes, permitiendo la reflexión, adquisición y fortaleza de atributos o cualidades para superar cualquier adversidad que se presente en la vida, siendo sujeto productivo dentro la sociedad. Según Imacaña (2018) la educación se sustenta en la Actualización y Fortalecimiento Curricular que forma parte del proyecto educativo nacional, tiene como fin orientar en el alcance de una educación de calidad, eficiente y productiva; sin embargo, la escasa relación entre el concepto de resiliencia, la comprensión lectora y el desarrollo de la escritura en contextos de vulnerabilidad dificulta el proceso de enseñanza y aprendizaje. Desde la perspectiva dinámica, interactiva y global del desarrollo humano, se resalta, además, que los niños y niñas que provienen de familias de nivel socio económico medio y bajo, no adquieren hábitos lectores; factor que influye en el cumplimiento de tareas, el bajo rendimiento, la escasa motivación y e insuficiente autoestima.

Según Becoña (2006) la resiliencia es el proceso de afrontamiento con eventos vitales desgarradores, estresantes o desafiantes de un modo que proporciona al individuo protección adicional y habilidades de afrontamiento que las que tenía previa a la ruptura que resulto desde el evento. Para Villalobos; Castelan (2016). El termino resilencia tiene su origen en el latín, resilio que significa volver atrás, volver de un salto, resaltar, rebotar; en educación la resiliencia caracteriza a aquellas personas que a pesar de nacer y vivir en situaciones de alto riesgo se desarrollan psicológicamente sanas y con éxito, sin consecuencias negativas 0 perturbadoras a largo plazo creando en ellos la propia autorregulación y no la imposición de 
normas externas de autoridad. Es un proceso de superación de la adversidad y de responsabilidad social. La tarea pedagógica es el diseño de acciones preventivas tanto individuales, grupales e institucionales, pero una prevención en donde la percepción de las personas sea concebida con recursos para desarrollar su propia resiliencia y ser un recurso para otro, capacidad de recuperarse, sobreponerse y adaptarse con éxito frente a la adversidad y de desarrollar competencia social, académica y vocacional pese a estar expuesto a acontecimientos adversos, al estrés grave o simplemente a las tensiones inherentes al mundo de hoy. La resilencia en la educación está concebida como un resorte moral, y se constituye en una cualidad de una persona que no se desanima, que no se deja abatir, que supera a pesar de la adversidad.

Para Villegas (2017) la resiliencia es la capacidad que tiene una persona para sobreponerse ante cualquier dificultad que se le presente en la vida y salir de la misma de manera airosa. El docente resiliente que es aquel que se ha recibido formación en torno a esta temática y que posee una serie de características personales (autoestima, seguridad, empatía, confianza, respeto etc.,) que lo hacen idóneo para trabajar la resiliencia en las aulas e inculcarlas a sus alumnos, ya que es imprescindible que un docente sea resiliente para trabajar a través de este tipo de metodologías. El alumno resiliente que es aquel que posee la capacidad de establecer unos objetivos y cumplirlos, sabe controlar y resolver conflictos, trabaja conjuntamente, expresa y maneja sus emociones, alumno es aquel que ha aprendido a través de estas metodologías, las cuales le están ayudando a fortalecer estos aspectos y resolver aquellas situaciones problemáticas que se le presentan en su día a día con éxito.
Para Flórez (2019) la palabra resiliencia está compuesta por el prefijo "re" y el verbo "salire" y la terminación "ia". El prefijo "re" indica intensidad e interacción de volver, recordar, rechazar y relatar. El verbo "salire" significa salir y saltar. Y el sufijo "ia" se refiere a la cualidad o modo de ser. La resiliencia se describe la capacidad que tiene un ser humano de superar las adversidades más complejas en su experiencia de vida, para volver a encontrarse en armonía consigo mismo y con los demás. De ahí que, se ha considerado como un modo de ser (actitud), capacidad (habilidad) que tiene cada persona para salir adelante de situaciones complejas de sus experiencias de vida. Por tanto, la resiliencia es asumida como la capacidad para resolver situaciones tensas y negativas que afectan a las personas, en el caso de los niños y las niñas influye en el desempeño escolar y su relación con los compañeros.

Según Gómez (2017), manifiesta que la resiliencia es un proceso relacionado con múltiples factores y que, en consecuencia, puede ser intervenida y fomentada. Se trata, por lo tanto, de una prueba clara de que es posible mejorar los logros de aprendizaje de quienes viven en contextos de vulnerabilidad. En la literatura, la resiliencia se entiende como una reacción positiva, un desarrollo constructivo o dinámico al enfrentarse a una catástrofe, amenaza o situación estresante. En el ámbito educativo, el concepto de resiliencia ha sido utilizado para describir diferentes fenómenos, tales como la adaptación a la escuela de los estudiantes pertenecientes a minorías étnicas, víctimas de abuso, o que han vivido situaciones traumáticas como la muerte de sus padres, guerra o desastres naturales. 


\section{Resiliencia educativa}

Para entender el significado de la resiliencia educativa se hondará en la historia de la resiliencia, pues su concepto es difundido al ser útil para quienes trabajan con niños y jóvenes. "Desde que surgió los traumas psíquicos, la conexión de las ideas exige que después de una descripción clínica y la investigación de las causas, por este motivo hay que tomar un rol activo en la prevención de traumas y velar por una buena reparación. Y para ello, se necesita conocer el concepto de resiliencia. El mismo nace y comienza a desarrollarse en el hemisferio norte, Rutter, en Inglaterra, Werner, en Estados Unidos, después se difundió a Europa, Francia, Países Bajos, Alemania y España, más tarde llegó a América Latina (Rodríguez, 2009). El término resiliencia a partir de los años 90 se lo conoció como Psicología positiva, dicho tipo se especializa en la indagación e investigación de todos los aspectos positivos que nos rodean y de este modo contemplarlos y tenerlos presentes para seguir progresando. En la actualidad, la resiliencia es la capacidad de una persona para sobreponerse ante situaciones desfavorables y continuar con éxito en su desarrollo integral (Villegas, 2017).
Existen varios cambios como el producto de la modernización y la globalización, demandan nuevas estrategias para afrontar los distintos factores adversos. Cada uno de ellos coexisten, interactúan y son influidos por una gama de condiciones, tanto internas o externas independiente de la persona, que desencadenan una cadena causal de comportamientos violentos. La resiliencia se ha caracterizado por ser un conjunto de procesos sociales y personales que posibilitan tener una vida sana a pesar de los factores internos o externos que se encuentren dentro de un contexto insano de una persona. Estos procesos tendrán lugar a través del tiempo dando paso a combinaciones positivas entre atributos de infantes y su medio familiar, social y cultural (Instituto de Investigación y Evaluación Educativa de la Universidad Pedagógica Nacional Francisco Morazán, 2013).

Se propone 4 categorías de factores resilientes que sirven para superar las adversidades que pueden afrontar los niños respectivamente con las características como se muestra en el cuadro.

Cuadro 1. Categorías de factores resilient

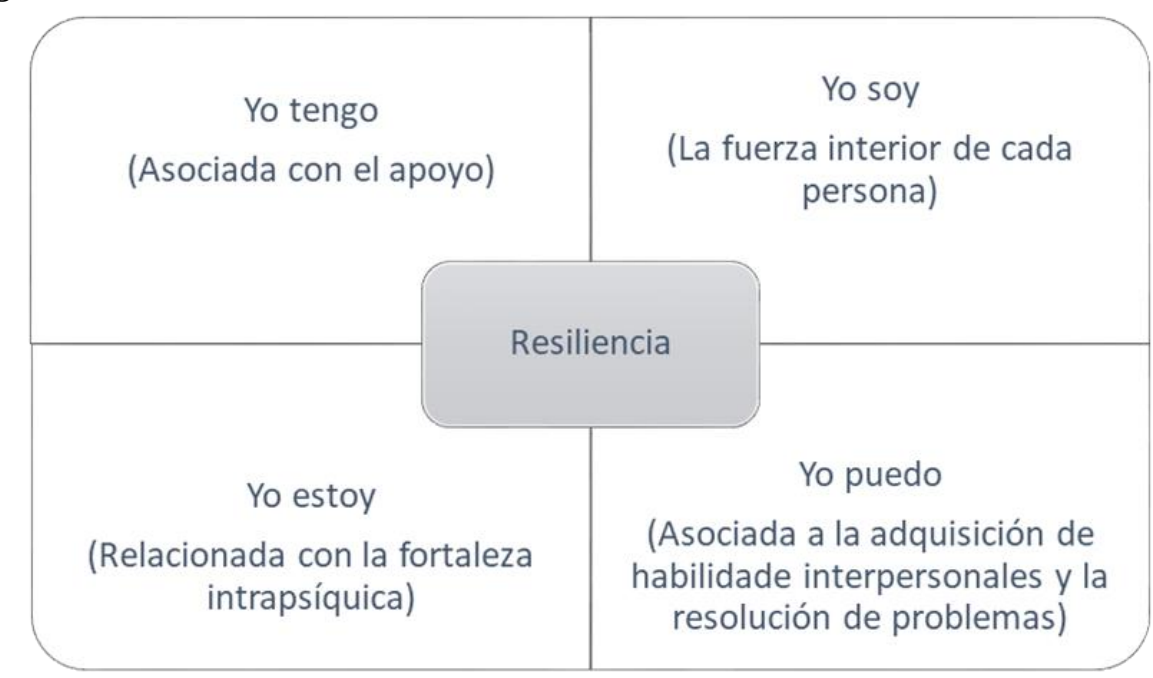


Descripción de El "Yo tengo"

Personas que tengo confianza y sé que me aman sin importar nada.

Personas que me ponen límites para que yo conozca cuando parar antes de estar en peligro o en problemas.

Personas que me enseñan cómo hacer las cosas de manera correcta por la forma en que lo hacen ellos.

Personas que quieren que aprenda por mi cuenta y de esta forma me desenvuelva solo. Personas que me cuidan y me ayudan cuando estoy enfermo, en peligro o necesito ayuda.

Descripción de "Yo soy"

Una persona que agrado a la gente y me quieren.

Me agrada hacer cosas lindas por los demás y mostrar mi preocupación.

Respetuoso conmigo mismo y con los demás.

Responsable de mis actos.

Seguro de que todo estará bien.

Descripción de "Yo puedo"

Conversar con las demás personas sobre lo que me asusta y lo que me molesta.

Encontrar la manera de resolver los problemas a los que me enfrento.

Controlarme cuando quiero realizar algo que está mal o es peligroso.

Averiguar el momento adecuado para dialogar con alguien o tomar medidas.

Encontrar a una persona para que me brinde su ayuda.

Yo estoy:

Dispuesto a responsabilizarme de mis actos.

Confiado de que las cosas estarán bien (Grotberg, 1999). 

vulnerabilidad de EGB. Media

\section{Tipos de resiliencia}

Existen diversos tipos de resiliencia que se encuentran dentro de diferentes contextos (Gobernación de Antioquia, 2015). Como se detalla en el cuadro 2.

Cuadro 2. Tipos de resiliencias

\section{Tipos de resiliencia}
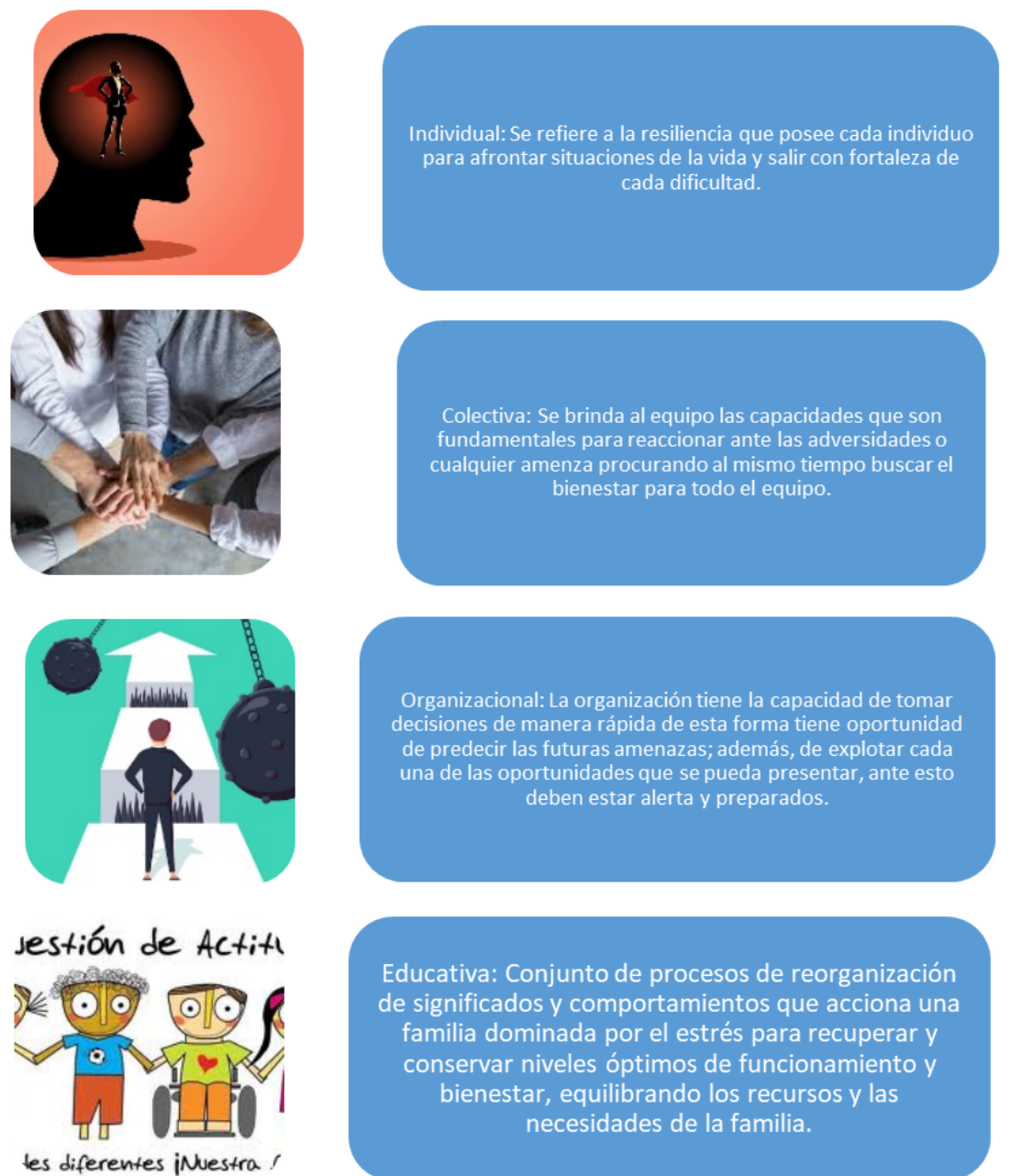

Educativa: Conjunto de procesos de reorganización de significados y comportamientos que acciona una

familia dominada por el estrés para recuperar y conservar niveles óptimos de funcionamiento y bienestar, equilibrando los recursos y las necesidades de la familia.

Familiar: Enlazada con el contexto educativo, es la capacidad de cada niño, adolescente o adulto de superar los problemas y retos que conlleva obtener resultados favorables dentro de un entorno hogareño no apto. 


\section{Vulnerabilidad}

La vulnerabilidad es definida como un proceso dinámico establecido por la interacción de los elementos que la componen, tales como edad, raza, etnia, pobreza, escolaridad, soporte social y presencia de agravantes a la salud. Vulnerabilidad es el riesgo que una persona, sistema $\mathrm{u}$ objeto puede sufrir frente a peligros inminentes, sean ellos desastres naturales, desigualdades económicas, políticas, sociales o culturales. Las vulnerabilidades adoptan diferentes formas, dependiendo de la naturaleza del objeto de estudio, sus causas y consecuencias. Frente a un desastre natural como un huracán, por ejemplo, la pobreza es un factor de vulnerabilidad que deja a las víctimas inmovilizadas sin capacidad de responder adecuadamente Nichiata; et.al., (2008).

\section{Niños con estado de vulnerabilidad}

Lloret; Monera; Bravo (2010) El niño es vulnerable porque se encuentra en riesgo constante de ser perjudicado, maltratado, perseguido, lastimado, discriminado, menospreciado o influenciado negativamente por agentes hostiles frente a los que se halla en situación de inferioridad, indefensión o fragilidad.

\section{¿Cuáles son los derechos de los niños en situaciones de vulnerabilidad? ¿Por qué son importantes?}

Los niños también disponen de todos los derechos humanos. No porque sean "los adultos del mañana" o "el futuro", sino porque a día de hoy son seres humanos. Al igual que otros grupos como las personas discapacitadas, las mujeres y las minorías étnicas, los niños pueden ser vulnerables, por eso tienen derechos específicos para protegerlos.
Además, algunos niños son especialmente vulnerables y tienen derechos adicionales que ayudan a garantizar que pueden vivir sus vidas de forma plena, con igualdad, dignidad y respeto. Estos niños se encuentran entre las personas más vulnerables del mundo debido a la violación de los derechos humanos, que incluyen la violencia y el abuso sexual, la explotación infantil y la negación de sus derechos civiles y políticos. El derecho a la no discriminación también es aquí un asunto importante, especialmente para las chicas y los grupos minoritarios.

Los niños discapacitados, los niños refugiados (especialmente aquellos que se encuentran solos) y los niños en conflicto con la ley son sólo ejemplos de niños a los que se les concede protección especial bajo la Convención sobre los Derechos del Niño y otros instrumentos internacionales sobre derechos humanos.

Debe recordarse que estos niños disponen de todos los derechos humanos, incluidos todos aquellos derechos que aparecen en la Convención sobre los Derechos del Niño, y no solo aquellos específicos a sus situaciones.

\section{Características de niños con vulnerabilidad}

Los agresores generalmente son conocidos de la familia y de los niños, ganan la confianza de todos y eso permite que los infantes no hablen. También tratan de mostrar y usar su poder de persuasión y buscan a niñas y niños que:

- Se sienten malos o estén devaluados por la familia.

- Necesiten protección y afecto.

- Que se les ha calificado de mentirosos o problemáticos.

- En los que los adultos cercanos no creen 
- Que han sufrido abusos de cualquier tipo.

- Inseguros, que manejan niveles mínimos de confianza con los adultos cuidadores primarios (padres y madres, tíos y cuidadores en casas hogar).

- Menores que han vivido o viven en aislamiento social, con poca convivencia y confianza con otras familias o cuidadores, porque el aislamiento no sólo comprende a las familias sino también a las personas resultado de la creciente movilidad y desintegración comunitarias.

- Cuando los niños o niñas presentan introversión y ensimismamiento.

- Presentan timidez.

- Viven descuido.

- Experimentan abandono emocional por parte de los padres o la familia.

- Presentan baja autoestima.

- Han desarrollado pocas habilidades para identificar y nombrar las emociones.

- Viven una dinámica familiar de comunicación sumisa y obediente, no asertiva.

- Presentan poca tolerancia a la frustración

Entre las causas que inciden en la existencia de vulnerabilidad, se menciona en primer término:

a) Falta de igualdad de oportunidades.

b) Incapacidad para satisfacer las necesidades.

c) Desnutrición y enfermedad.

d) Incapacidad para acceder a los servicios públicos.

e) Marginación.

\section{Características de la vulnerabilidad}

Se puede decir que, la vulnerabilidad se manifiesta de tres formas:

Multidimensional. Porque se manifiesta tanto en distintas personas como en grupos determinados o identificados por características comunes, así como en comunidades, objetos en los que la vulnerabilidad se manifiesta de diferentes formas y a su vez en diversas modalidades.

Integral. Porque la existencia de la misma, por cualquiera de las causas que le dan origen, implica la afectación no de uno sino de varios de los aspectos de la vida de las personas que la sufren.

Progresiva. Debido a que como una situación lleva a la otra, como en un efecto dominó, ésta se acumula y aumenta en intensidad, lo que provoca consecuencias cada vez más graves en la vida de quienes sufren de alguna causa de vulnerabilidad $y$ sus consecuencias, lo que propicia el surgimiento de nuevos problemas y a un aumento de la gravedad de la vulnerabilidad, por lo que esta condición se vuelve cíclica.

La vulnerabilidad provoca que, a nivel jurídico, quienes la sufren sólo vean reconocidos sus derechos y libertades fundamentales a un nivel formal, ya que en la realidad o en el mundo material es en donde se les limitan, nulifican o desconocen, debido a prácticas culturales, sociales, políticas o económicas, movidas por intereses diversos en los que el poder y el abuso del mismo tienen una influencia importante; es decir, no existen las condiciones para su goce y ejercicio.

Las investigaciones previas han permitido reconocer los distintos estados de vulnerabilidad por las cuales un cierto número de infantes puedan padecer entendiendo que pueden ser la exposición a contingencias y tensión, y la dificultad al afrontarlas. Existen factores internos (edad, género, discapacidad, etc.) y externos (contexto social, empleo, situación económica, etc.), la vulnerabilidad al ser multifactorial puede colocar a una persona en muchas situaciones que a continuación se detallan Lara (2013) algunas de ellas son: 
Vulnerabilidad educativa. Relacionada con la situación educativa de los padres, analfabetismo o solo han cursado los primeros grados de educación.

Vulnerabilidad habitacional. Se refiere a las condiciones de la vivienda.

Vulnerabilidad laboral. Tiene relación a las condiciones laborales de los progenitores.

Vulnerabilidad familiar. Relacionado con las condiciones de fragilidad en los lazos familiares (González y Labandal, 2018). La vulnerabilidad puede crear distintas maneras de comportamientos y conductas en los infantes que si no existe una correcta intervención continuara hasta su adultez. Pueden crear, desconfianza, violencia, desesperanza, frustración, resistencia a la autoridad, baja autoestima y valorización personal (Dirección General de Coordinación Operativa y Capacitación, 2011).

Por esto motivo, es importante interceder para que los niños y adolescentes se reconozcan como víctimas de maltrato. La toma de conciencia por los malos tratos, resultado de los factores internos y externos. Los niños tienen el derecho a saber $\mathrm{y}$ comprender que ellos no son culpables de las adversidades que perecen o de las malas condiciones de su entorno (Saavedra, et. al., 2015).

\section{Resiliencia y su influencia en la escolaridad}

Ahora, la resiliencia educativa es la capacidad de resistir, la fortaleza interna, como lo entienden los franceses "courage" para afrontar todas las circunstancias de la vida personal, familiar, profesional y social. Es necesario que cada infante explore las fuentes de su propia fortaleza personal, considerando que la perfección de la fortaleza es la constancia, la capacidad de acometer y sobrellevar. El proceso de adquirir y desarrollar la resiliencia es de hecho transcurso de la vida, porque cada niño debe superar episodios de estrés, traumas y rupturas es parte de vivir, sin quedar marcado de por vida y ser feliz a pesar de las adversidades. De esta forma resulta concurrente con la Pedagogía al reconocerse que es la ciencia que permite la enseñanza de vivir una buena vida, aceptando el sufrimiento que conlleva (Villalobos y Castelán, 2016).

En la actualidad gran parte de los infantes $\mathrm{y}$ adolescentes se encuentra en un contexto que no es considerado apto para el desarrollo por lo cual escuela y docentes deben estar preparados para trabajar y afrontar todo aquello que lleve a cuestas el alumnado. Para que el estudiante pueda progresar y sepa controlar determinadas situaciones es pertinente que el docente tenga conocimiento sobre metodologías y pedagogías que favorezcan el desarrollo integral del educando. La escuela se esmera por identificar con premura las dificultades de los educandos que pueden presentar signos de vulnerabilidad, inadaptación y fracaso escolar para consecutivamente, implementar programas con soluciones específicas como: adaptaciones curriculares, compensación de déficits, intervención en problemáticas, prevención de conductas riesgosas, entre otras (Uriarte, 2006)

Estos programas reconocen que los docentes aún no se encuentran totalmente capacitados para hacer frente a la enseñanza eficiente de los niños, quienes atraviesan por una gama de dificultades cuando tienen que incorporarse a una nueva forma de trabajo al recorrer un nivel educativo a otro: además, desenvolver un conjunto de habilidades y capacidades que suponen ya han sido previamente desarrolladas. El papel del 
docente, sus saberes y las creencias que posee acerca del desarrollo de las habilidades fundamentales para la lectoescritura, pueden explicar las formas estrictas y tradicionales de enseñanza de la lectoescritura situando a algunos niños con estilos diferentes de aprendizaje en una clara posición de desventaja incluyendo los factores externos (López, Ramos, y Mancilla, 2009).

La complejidad en la adquisición de la lectoescritura es a partir de la revisión de las áreas en las que tienen problemas los niños y no logran los propósitos establecidos y manifiestan cierto grado de discrepancia con el resto de los compañeros de grupo al no poder desenvolverse en las tareas relativas a la correspondencia entre lo hablado y lo escrito.

La resiliencia se verá afectada por la relación de los educandos con el contenido académico. Como nos referimos al proceso de la lectoescritura se pretende fortalecer tres aspectos el fonológico, el sintáctico y el semántico, que junto con las experiencias establecen bases para que los aprendices doten de sentido a este proceso que se privilegia en el ámbito escolar al igual que la resiliencia, ya que ambos comprometen aspectos cognitivos y no cognitivos (Gómez, y Rivas, 2017).

El dominio de la lectoescritura como herramienta del conocimiento es reconocido tanto social como individualmente afirmando que puede tratarse hasta de una conducta que forma parte de la psicología del individuo desde su infancia hasta la adultez y constituye un punto clave en su comunicación. El impulso de la resiliencia desde la escuela es parte del proceso educativo y más como una ventaja para el desarrollo de la lectoescritura y ayudara al estudiante a afrontar las diversas dificultades que encuentre en el proceso ya sean factores internos $o$ factores externos (Linuesa, 2011).

\section{REFERENCIAS}

Alcívar, D. (2013). La lecto-escritura y su incidencia en el rendimiento escolar de los estudiantes del quinto grado del centro de educación básica Pedro Bouguer de la parroquia Yaruquí, cantón Quito, provincia de Pichincha. Quito, Pichincha, Ecuador: Universidad Técnica de Ambato

Arias, A. (2017). Técnicas de expresión plástica e inteligencia emocional. Estudio en niños de la "Fundación Jardín del Edén". SalcedoCotopaxi 2016. Latacunga: Universidad Nacional de Chimborazo

Barros. (9 de Noviembre de 2017). ¿Cómo fortalecer la autoestima y la comprensión lectora? Revista Vinculando, 10

Becoña, E. (2006). Resiliencia: definición, características y utilidad del concepto. Psicopatología y Psicología Clínica, 125146

Caiza, L., Morales, J., y Barragán, L. (12 de Septiembre de 2018). Enfoque de la residencia en las instituciones educativas a nivel de educación básica ante desastres naturales en la ciudad de San Miguel Ecuador. Revista de Ciencias de Seguridad y Defensa, 22. Obtenido de http://geo1.espe.edu.ec/wpcontent/uploads/2018/10/10.pdf

Camacho, M., Rodríguez, L., Gracia, D., y Sarmiento, A. (2018). Didáctica de la lectoescritura en experiencias de resiliencia: un proceso consciente. Innovaciones educativas para favorecer los de lectoescritura. Barranquilla, Colombia: Universidad Simón Bolívar

Dirección General de Coordinación Operativa y Capacitación. (10 de Octubre de 2011). La situación de vulnerabilidad social de la infancia y la adolescencia. Obtenido de http://institutocienciashumanas.com/wpcontent/uploads/2020/03/Material-deapoyo-para-capacitacion-docente-lavulnerabilidad-social-de-la-infancia-y-laadolescencia.pdf

Eusse, P., y González, S. (2018). Leo, escribo, me fortalezco: la lectoescritura como potenciadora de la resiliencia en niñas, 
niños y adolescentes que han pasado por situaciones de vulnerabilidad en la Fundación Casa Hogar de las Merced. Medellín, Antioquia, Colombia: Universidad de Antioquia.

Flores, Y., y Marcillo, C. (18 de Julio de 2020). Lecturas motivadoras para fortalecer la resiliencia en los estudiantes de Educación General. Dominio de las Ciencias, 6(3), 832851. Ciencias de la Educación, 6(3), 20. doi:http://dx.doi.org/10.23857/dc.v6i3.132 1

Flórez, M. Y. (2019). La capacidad de resiliencia de los niños y niñas de 8 años, en la Unidad. Quito: Universidad Andina Simón Bolívar

Gobernación de Antioquia. (18 de Mayo de 2015). Resiliencia. Obtenido de https://www.antioquiatic.edu.co/images/di gitalmente/pdf/resiliencia.pdf

Gómez, G. (2017). Resiliencia académica nuevas perspectivas de interpretación de la aprendizaje en contextos de vulnerabilidad social. Universidad de O'Higgins, 13. Obtenido de file:///C:/Users/PC146/Downloads/Resiliencia_Conf.pdf

Gómez, G., y Rivas, M. (2017). Resiliencia académica, nuevas perspectivas de interpretación del aprendizaje en contextos de vulnerabilidad social. Calidad en la Educación, 215-233. Obtenido de https://scielo.conicyt.cl/pdf/caledu/n47/0 718-4565-caledu-47-00215.pdf

González, D., y Labandal, L. (20 de Febrero de 2018). La infancia en contextos de vulnerabilidad: la educación como apuesta al futuro. Obtenido de https://feeye.uncuyo.edu.ar/web/X-CNREDUEI/eje2/Gonzalez.pdf

Grotberg, E. (1999). Early Childhood Development: Practice and Reflections. Alabama: Bernard van Leer Foundation. Obtenido de https://bibalex.org/baifa/Attachment/Doc uments/115519.pdf

Instituto de Investigación y Evaluación Educativa de la Universidad Pedagógica Nacional Francisco Morazán. (11 de Marzo de 2013). Resiliencia educativa de estudiantes ante los riesgos sociales generados por las barras juveniles de fútbol en Honduras. Obtenido de http://wbgfiles.worldbank.org/documents/ hdn/ed/saber/supporting_doc/CountryRep orts/EDR/RES_Research\%20Local\%20Hon duras\%20futbol_SP.pdf

Imacaña, L. (2018). Estrategia lúdica para la enseñanza de Matemática en Educación General Básica Elemental en la Unidad Educativa Intercultural Bilingüe Tamboloma. Ambato, Ecuador: Pontificia Universidad Católica del Ecuador. Obtenido de

https://repositorio.pucesa.edu.ec/bitstrea $\mathrm{m} / 123456789 / 2418 / 1 / 76684 . p d f$

Lara, D. (2013). Grupos en situación de vulnerabilidad. Derechos Humanos, 1-78. Obtenido de http://appweb.cndh.org.mx/biblioteca/arc hivos/pdfs/fas_CTDH_GruposVulnerabilida d1aReimpr.pdf

Linuesa, M. (9 de Septiembre de 2011). El aprendizaje de la lectoescritura. Aspectos de su problemática y algunas perspectivas actuales. Obtenido de http://espacio.uned.es/fez/eserv/bibliuned:20270/ aprendizaje_lectoescritura.pdf

Lloret, F. T., Olmos, C. E., y Pastor, B. M. (2010). Vulnerabilidad Infantil. Madrid

López, M., Ramos, R., y Mancilla, M. (17 de Julio de 2009). La adquisición de la lectoescritura en educación básica desde las teorías interaccionistas sujeto-ambiente. El caso de tres escuelas primarias de Tuxtla Gutiérrez, Chiapas. Obtenido de http://comie.org.mx/congreso/memoriaele ctronica/v10/pdf/area_tematica_01/ponen cias/1055-F.pdf

Mora, N. (2018). La adquisición de la lectoescritura en el proceso de enseñanzaaprendizaje de los niños y niñas de segundo año de Educación General Básica de la Unidad Educativa "Alicia Marcuard de Yerovi” del Cantón Salcedo. Latacunga, Cotopaxi, Ecuador: Universidad Técnica de Ambato 
Nichiata, L. Y., Bertolozzi, M. R., Takahash, R. F., y Fracoll, L. A. (2008). L a utilización del conepto "vulnerabilidad" por enfermería. Latino-am Enfermagem, 28-42

Ortíz, M. (2016). Comprensión lectora y las capacidades lingüísticas de los estudiantes de séptimo año de educación básica. Ambato, Ecuador: Universidad Técnica de Ambato.

Pérez, s. (Enero de 2016). "Material didáctico para la iniciación de la lectura. Obtenido de http://recursosbiblio.url.edu.gt/tesiseortiz /2016/05/84/Perez-Skarltt.pdf

Pinargote, E., Cedeño, H., y Romero, G. (2018). Importancia del fomento de la resiliencia en el proceso de la enseñanza aprendizaje con estudiantes con discapacidad. Atlante Cuadernos de Educación y Desarrollo.

Rivas, G. G. (2017). Resiliencia académica, nuevas perspectivas de interpretación de aprendizaje en contextos de vulnerabilidad. CALIDAD EN LA EDUCACIÓN, 215-233

Rodríguez, A. (2009). Resiliencia. Revista Psicopedagogía, 291-302. Obtenido de http://pepsic.bvsalud.org/pdf/psicoped/v 26n80/v26n80a14.pdf

Saavedra, E., Salas, G., Cornejo, C., y Morales, P. (2015). Resiliencia y calidad de vida. Chile:
Universidad Católica de Maule. Obtenido de https://www.academia.edu/30917204/LI BRO_RESILIENCIA_pdf

Tomasini, G. (2012). Resiliencia en educación especial: Una experiencia en la escuela regular. . Zaragoza, España: Editorial Gedisa.

Uriarte, J. (2006). Construir la resiliencia en la escuela. Revista de Psicodidáctica, 7-24. Obtenido de https://addi.ehu.es/bitstream/handle/108 10/7215/Rev.\%20Psicodidactica $\% 2011 \%$ $281 \% 29 \% 20-\% 207-$ 24.pdf?sequence $=1$ yisAllowed $=y$

Villalobo Torres, E. M., y Castelán García, E. (2016). La resilencia en la Educación. México: Cepindalo.

Villalobos, E., y Castelán, E. (2016). La resiliencia en la educación. CEPIndalo, 1$10 . \quad$ Obtenido de http://educespecialjujuy.xara.hosting/inde x_htm_files/RESILENCIA\%20EN\%20LA\%2 OEDUCACION.pdf

Villegas, A. (10 de Marzo de 2017). Resiliencia Educativa. Obtenido de https://www.eumed.net/librosgratis/actas/2017/educacion/59resiliencia-educativa.pdf 